\title{
Numerical method for analysis of the correlation between ferrofluid optical transmission and its intrinsic properties
}

\author{
Á. Sanz-Felipe*, J.C. Martín \\ Departamento de Física Aplicada and I3A, Universidad de Zaragoza, Pedro Cerbuna 12, 50009 Zaragoza, Spain
}

\section{A R T ICLE INFO}

\section{Keywords:}

Ferrofluid simulation

Magneto-optics

Mixture law

Ferrofluid intrinsic parameters

\begin{abstract}
A B S T R A C T
A numerical method to simulate the ferrofluid particle distribution evolution is presented. Also, the optical transmission of the distributions obtained is calculated by two numerical methods. The first one consists on a numerical propagation of an electromagnetic wave through the sample. The second one analyzes the aggregates' mean length to obtain the optical transmission through a mixture law. As an illustration of the possibilities of the method developed, it is applied to analyze how ferrofluid optical transmission changes after magnetic field application depend on intrinsic properties of the colloid such as its nanoparticle concentration and surfactant repulsion represented by means of the final distances between consecutive particles forming chains. Changes in the attenuation factor of these samples show the trends expected from the Literature.
\end{abstract}

\section{Introduction}

Ferrofluids or magnetic fluids are interesting materials due to their range of potential applications [1]: rheological and tribological (damping, heat dissipation), biomedical (drug targeting or hyperthermia for cancer therapy, magnetic separation of cells), etc. The capability of tuning their optical properties by means of an external magnetic field makes them also attractive for photonic applications such as switching, modulation and all-optical magnetic sensing and transmission controlling [2-4]. Many experimental results relating ferrofluid transmission optical changes and applied magnetic field have been reported [5-9]. However, there is a lack of a generalized model capable of predicting this behavior quantitatively starting from the intrinsic characteristics of a particular ferrofluid. Several simulations [10-12] and theoretical models [13-17] have also been presented in the Literature with the aim of finding a simplified treatment of the phenomenon in both ways. For instance, there are models based on the analysis of the aggregation scattering $[8,15]$, models centered in the dependence on concentration [16] or size [17], etc. These models are shown to describe correctly experimental measurements on a sample or a family of samples, but they contain fitting parameters which can only be experimentally determined. Therefore, they cannot be employed to anticipate the magneto-optical properties of another sample prior to any measurement.
The aim of this work is the proposal of a numerical model capable of predicting the magneto-optical response of a ferrofluid departing from its intrinsic properties. Obviously, the choice of the optimal ferrofluid for any potential magneto-optical device would be much easier counting on a model like this. Besides, a complete model would allow one to design ferrofluids with customized magneto-optical properties, which is the key point to be discovered yet. As shown in the Literature, the difficulty in modeling these substances is due to the complexity of the phenomena involved: magnetic forces that give rise to nanoparticles attraction and coalescence into chains $[18,19]$, leading to aggregates of different shapes whose interaction with light constitutes a demanding scattering problem $[10,20,21]$. Furthermore, Brownian motion and surfactant stabilization have to be taken into account. This combination involves a huge number of parameters which determine the intensity of the different physical interactions in a very complex way since many of the parameters are somehow unknown.

Specifically, in this paper we present a numerical method oriented to predict the changes in optical transmission of a ferrofluid caused by its exposure to an external magnetic field. The calculation presents two stages. The first one solves the temporal evolution of the nanoparticles' locations, velocities and dipoles' orientation depending on the ferrofluid intrinsic conditions. The main physical interactions between the nanoparticles are considered: dipole-dipole forces, external and internal magnetic fields and surfactant stabilization. The second section cal-

\footnotetext{
* Corresponding author.

Email address: angel_sf@unizar.es (Á. Sanz-Felipe)
} 

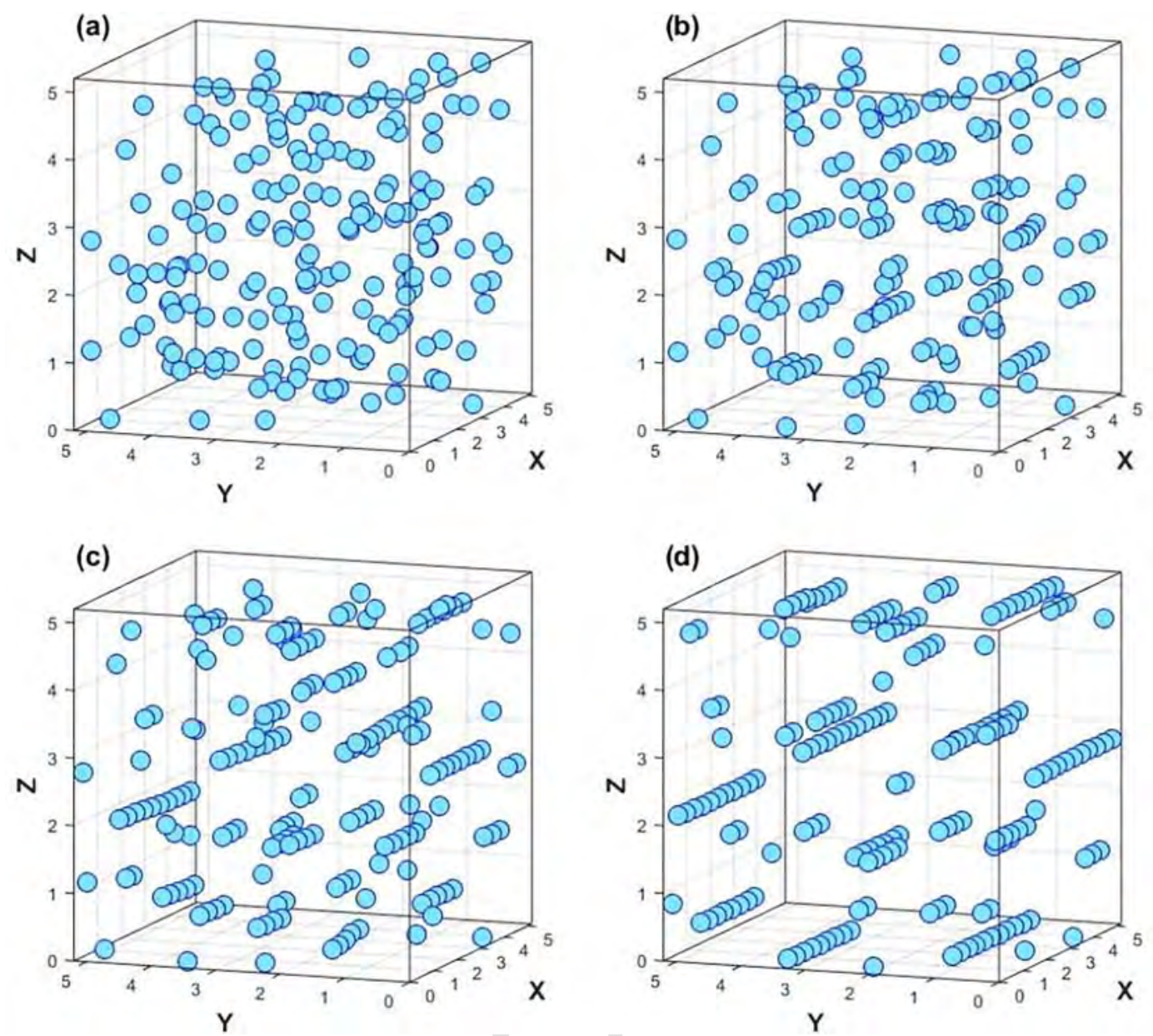

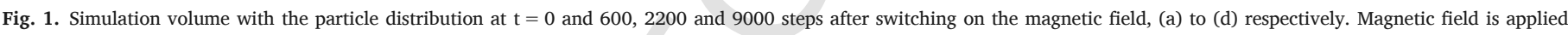
along X direction. All the dimensions are set in $\mu \mathrm{m}$.
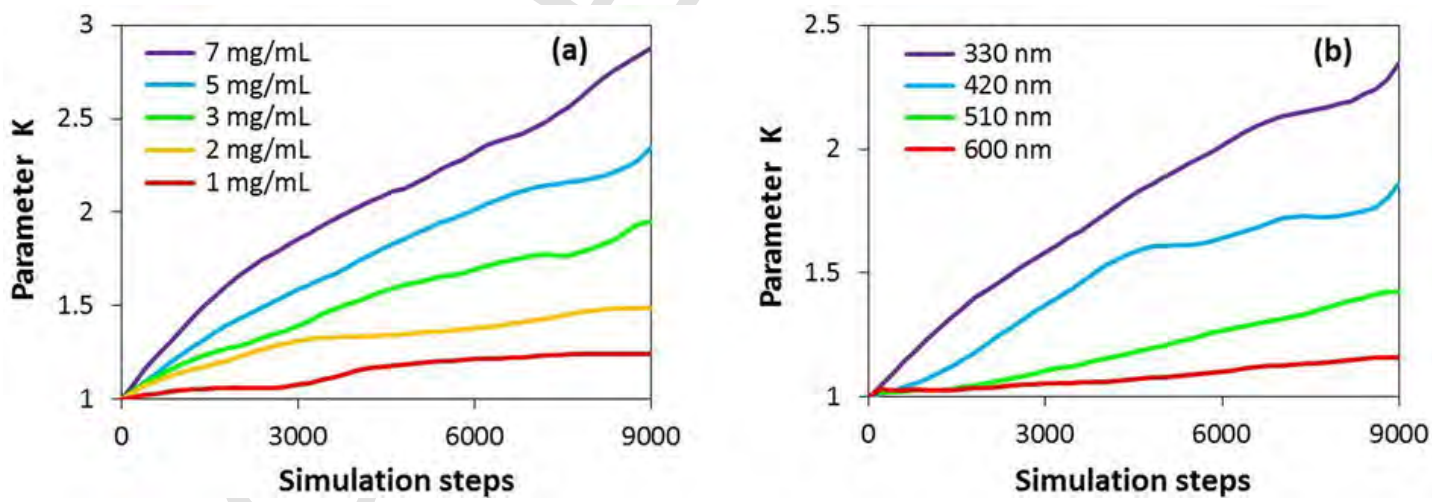

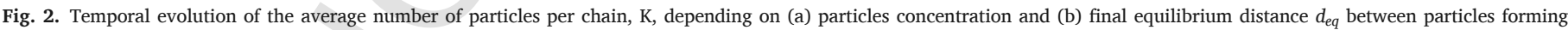
chains.

culates the sample optical transmission by means of two methods that prove to be complementary: on the one hand, by numerical propagation of an electromagnetic wave over the particles' distributions and, on the other hand, by means of a mixture law $[22,23]$. This way, a general method is proposed to obtain the magneto-optical response of any ferrofluid from its intrinsic parameters. The method will also be useful for analytic description of these substances in order to optimize a sample design, face to their magneto-optic applications.
The paper is organized as follows. Section 2 is devoted to describe our ferrofluid evolution simulation and the analysis of the chains length. Section 3 presents the calculation of the optical transmission by two methods: the first one shows the results of numerical propagation of an electromagnetic wave through the simulation volume, and the second one presents the application of the simulated chains length into a mixture law. Section 4 presents the conclusions. 

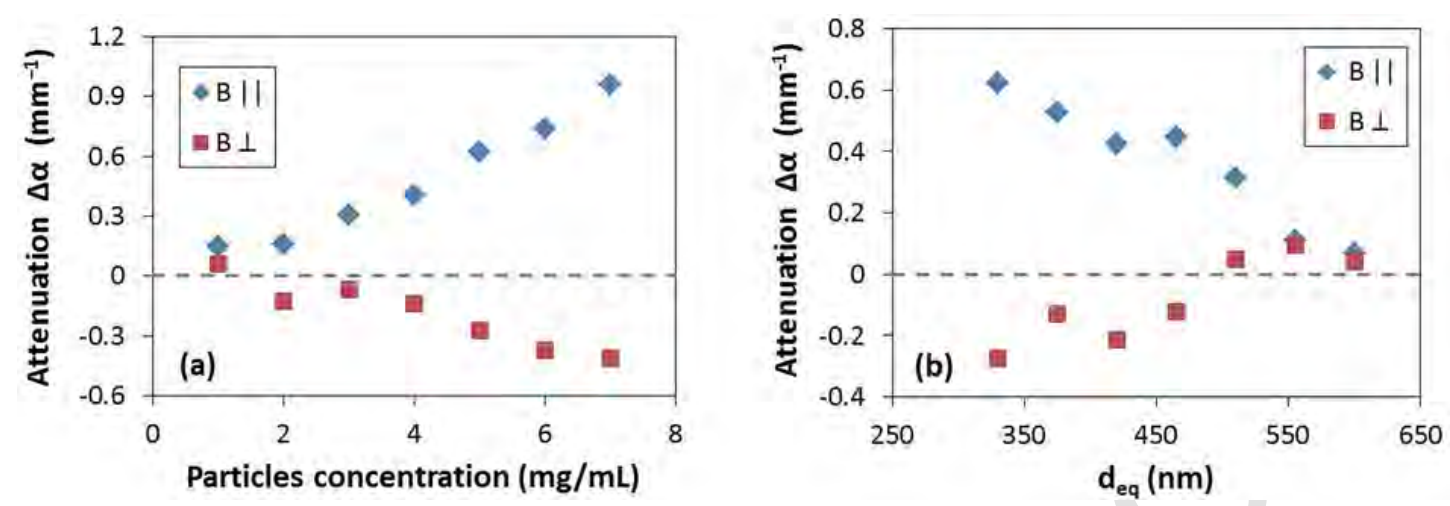

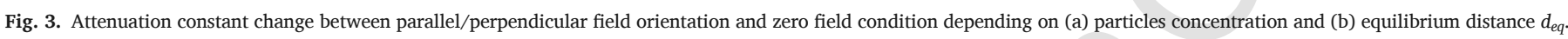
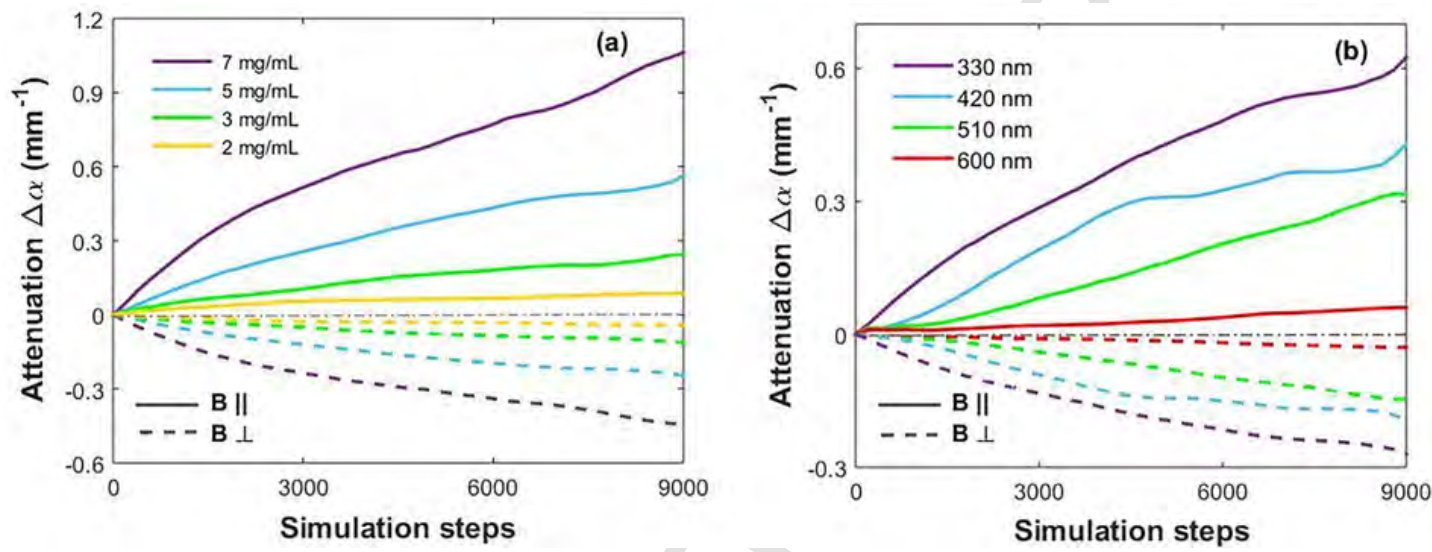

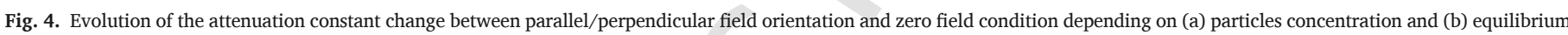
distance $d_{e q}$.

\section{Formation of chains}

\subsection{Numerical method}

A computer program has been developed which simulates how the nanoparticle distribution present in a ferrofluid evolves after the applied magnetic field has been modified. In particular, in this work we focus on the evolution of an initially random particle distribution, corresponding to absence of external magnetic field, since the instant $t=0$ in which an external magnetic field is switched on. The nanoparticles' locations, velocities and dipoles' orientation are calculated at different time intervals separated by a certain time step $\Delta \mathrm{t}$. The main physical interactions between particles are considered: dipole-dipole forces, surfactant stabilization and momentum caused by the external magnetic field. All of them lead to translation and rotation movements every temporal step along the simulation. The magnitude of these interactions are determined by the intrinsic properties of the ferrofluid: each nanoparticle is treated as a magnetic dipole whose moment is determined by the magnetization and average particle size of the sample, the surfactant quantity determines the equilibrium distance between particles and the fluid viscosity gives rise to greater or lower friction.

However, some well-known effects have not been taken into account for the sake of saving computation time:

- Thermal effects are not considered; their main effect is slowing down the system evolution (by both rotational and translational Brownian dispersion) towards the configuration of minimum free energy [14], so they would be essential if the aim was describing the characteristic time of these processes. Nevertheless, as the calculation time in- creases proportionally to the number of steps, this aim cannot be afforded unless the computation time becomes highly multiplied. Apart from a certain statistical deviation from the main trend, considering thermal effects or not does not modify the fundamental processes that take place in the system, it only implies that these processes take place at a faster velocity than in real samples Therefore, the time scale is not considered in our results.

- The model proposed treats interactions exclusively as the sum of the interactions between pairs, calculated considering a surrounding non-magnetic medium, neglecting that nearby particles modify the magnetic field lines. Van der Waals force has been neglected as no important effects have been seen on our calculations: for particle-particle interaction, dipole-dipole forces dominate at short distances while Van der Waals forces are predominant only at long distances, situation in which all forces are negligible. This way, our calculation procedure explains correctly chain formation but not chain coalescence, which would require a much more involved treatment taking into account the above-mentioned effects.

- All particles considered have the same size. Of course real samples present a distribution of particle sizes. Often the fabrication process frequently guarantees a low statistical dispersion, so that its effect is negligible. In cases of higher dispersion, the number of particles for the simulation is much higher than for uniform size particles, in order to get a statistically significant distribution. As computation times depend exponentially on particle number, we do not consider worthwhile to account for this detail.

The interparticle interactions are governed at large particle distances by the dipole-dipole force. A particle i with magnetic momentum $\vec{m}_{i}$ will interact with a second particle $\mathrm{j}$ located at a distance 
$r_{i j}=\left|\vec{r}_{j}-\vec{r}_{i}\right|$ according to the following expression [24]:

$$
\begin{aligned}
& \vec{F}_{i j}=\frac{3 \mu_{0}}{4 \pi r_{i j}^{5}}\left[\left(\vec{m}_{i} \bullet \vec{r}_{i j}\right) \vec{m}_{j}+\left(\vec{m}_{j} \bullet \vec{r}_{i j}\right) \vec{m}_{i}+\left(\vec{m}_{i} \bullet \vec{m}_{j}\right) \vec{r}_{i j}\right. \\
& \left.-\frac{5\left(\vec{m}_{i} \cdot \vec{r}_{i j}\right)\left(\vec{m}_{j} \cdot \vec{r}_{i j}\right)}{r_{i j}^{2}} \vec{r}_{i j}\right]
\end{aligned}
$$

The particles are coated by a polymeric surfactant layer which stabilizes them in the carrier fluid preventing them from aggregation. In order to model its effect in a simple way, we propose a repulsive interaction (Eq. (2)). The parameter $d_{\text {repul }}$ represents the distance where a sort of potential barrier is located, $n$ is a number that describes the dependence of this force on the distance, and $\mathrm{q}$ is a constant which determines the strength of the surfactant repulsion.

$\vec{F}_{\text {surf }, i j}=\frac{q}{\left(r_{i j}-d_{\text {repul }}\right)^{n}} \vec{r}_{i j}$

Since this force is somehow unknown, we can select these values in order to obtain a realistic behavior of the ferrofluid compared to the effects already seen at the laboratory. The equilibrium between dipole-dipole force and surfactant repulsion determines the distance $d_{e q}$ between two consecutive particles in a stable chain. The distance $d_{e q}$ can be tuned by choosing the values of the different parameters in Eq. (2).

Of course, a rotation caused by the presence of the external magnetic field is included. The magnetic field caused by the particle dipoles is rather weaker than the external one. For that reason, since this work is only devoted to analyze the sample with the field switched on, torque on the particle $\mathrm{i}, \tau_{\mathrm{i}}$, is calculated considering only the external magnetic field $\mathrm{B}_{\text {ext }}$ :

$\vec{\tau}_{i}=\vec{m}_{i} \times \vec{B}_{\text {ext }}$

Finally, friction terms proportional to the linear and angular velocities of each particle are also considered. They are calculated taking into account the solvent viscosity and treating particles as spheres [25].

Each particle is characterized by its location, dipole orientation, linear and angular velocity. Before external magnetic field switch on, we consider that particles do not move. At $\mathrm{t}=0$ (switch on time), linear and angular accelerations are calculated. With these data, the state of each particle at $\mathrm{t}=\Delta \mathrm{t}$ is calculated. Next, linear and angular acceleration of each particle at $\mathrm{t}=\Delta \mathrm{t}$ is calculated, which allow one to obtain the state of each particle at $t=2 \Delta t$, and so on.

\subsection{Results}

Fig. 1 illustrates the particle distribution of a ferrofluid before magnetic field switch on and also at three instants after switch on. A ferrofluid composed of magnetite nanoparticles with water as solvent is considered. Nanoparticles are spheres with a core of radius $50 \mathrm{~nm}$ (PdI $=0.176$ so we will consider them as monodisperse), magnetization $30 \mathrm{~A} \mathrm{~m}^{2} \mathrm{~kg}^{-1}$, and a surfactant layer (58 wt\% MMA and $42 \mathrm{wt} \%$ EGDMA) up to a total radius $\mathrm{R}=92 \mathrm{~nm}$. These properties correspond to MagP ${ }^{\circledR}$ nanoparticles, manufactured by NanoMyP. The magnetite density used is $5170 \mathrm{~kg} \mathrm{~m}^{-3}$ while the polymeric density is $983.6 \mathrm{~kg} \mathrm{~m}^{-3}$ $[26,27]$. Simulation in Fig. 1 is an example carried out with 180 particles within a cube whose edge length is $5.2 \mu \mathrm{m}$. The external magnetic field considered is $71.3 \mathrm{G}$ and translational and rotational damping coefficients employed are $1.73 \times 10^{-9} \mathrm{~s}^{-1}$. Using $\mathrm{n}=3$ and $d_{\text {repul }}=3 R$, we select the parameter $d_{e q}$ to take the value of $330 \mathrm{~nm}$. The simulation step time is $\Delta \mathrm{t}=10 \mu \mathrm{s}$.

Obviously, the visual inspection at different time instants gives valuable insight of the process but it is awkward to deal with such information. For this reason, we prefer to characterize each state of a nanoparticle set by the parameter $\mathrm{K}$, defined as the average number of particles per chain (isolated particles are counted as chains of one particle). The parameter $\mathrm{K}$ can be computed at each calculation time step. Fig. 2 shows how the parameter $\mathrm{K}$ evolves along time as a function of nanoparticles concentration (Fig. 2(a)) and as a function of $d_{e q}$ (Fig. 2(b)). Both simulations have been carried out with parameters $n=4$ and $d_{\text {repul }}=3 R$. Simulations in Fig. 2(a) uses a $d_{e q}=330 \mathrm{~nm}$, while concentration in Fig. 2(b) is $\varphi=5 \mathrm{mg} / \mathrm{mL}$. As expected, high concentrations and low $d_{e q}$ values favor particle aggregation.

\section{Optical transmission}

Beyond the capability of the chain formation process description, our objective is to obtain how the optical transmission of the ferrofluid evolves along this process. Specifically, in order to characterize this property we make use of the attenuation constant $\alpha$ defined as usually through $\mathrm{I}(\mathrm{x})=\mathrm{I}(0) \mathrm{e}^{-\alpha \mathrm{x}}$, where $\mathrm{x}$ is the coordinate in the propagation direction and $\mathrm{I}(\mathrm{x})$ is the light intensity at the $\times$ coordinate. For the sake of clarity, we remind that $\alpha$ is related to the imaginary part of the complex refractive index, $n^{*}=n-\kappa i$, by means of $\alpha=4 \pi \kappa / \lambda_{0}$, where $\lambda_{0}$ is the radiation wavelength in vacuum.

\subsection{Calculation by electromagnetic wave propagation}

The attenuation constant $\alpha$ of any cubic sample similar to the ones described before can be carried out numerically by means of a software capable of simulating electromagnetic wave propagation through a heterogeneous medium. Here, the numerical tool BeamPROP from RSoft CAD has been employed to propagate a plane wave. In order to do so, the cubic cell of $5.2 \mu \mathrm{m}$ generated by the procedure described in Section 2 can be taken at any given time instant along the process of chain formation. This cell is replicated to form a calculation domain of $30 \times 30 \times 88.4 \mu \mathrm{m}^{3}$ composed of stacked and concatenated copies of the original cell avoiding any possible pattern. The longest side is oriented in the propagation direction considered. According to our trials, this dimension is necessary to get an adequate precision in the determination of $\alpha$. Transversal extension is also necessary to reduce diffraction effects.

We use the normalized transmission $\mathrm{T}_{\text {norm }}$ defined as the absolute transmission after magnetic field application divided by the absolute transmission at the initial state. This way, we obtain a change in attenuation constant $\Delta \alpha$ defined by $\Delta \alpha=\alpha_{\|, \perp}-\alpha_{0}=-\ln \left(T_{\text {norm })} / x\right.$. Fig. 3 shows changes in attenuation constant between the initial and final states in the different cases shown in Fig. 2. The working wavelength is $632.8 \mathrm{~nm}$ (He-Ne laser). The refractive indexes considered are: 1.3319 for the solvent (water) [28], 2.3602-0.1472i for the nanoparticle magnetite core [29] and 1.43 for the surfactant $[26,27]$.

The trends obtained in Fig. 3 are in agreement with experimental results [23]: parallel and perpendicular field applications produce an increase and decrease on the attenuation constant respectively, and therefore, a loss in transmission in parallel configuration and an increase in the perpendicular one. Furthermore, it is logical to expect that, the higher the particle concentration, the bigger the change in transmission (Fig. 3(a)): particles are closer to interact with each other and form longer chains. On the other hand, the lower the distance between particles in a chain, the less the change in transmission (Fig. 3(b)): if the distance between particles grows, scattering becomes more individual than collective of each chain. Concerning Fig. 3(b), it is logical that the trend is more irregular as the scattering effects are highly dependents on distances between scatterers, and more simulations would be needed to improve the statistics.

Summing up, usefulness of the calculation method is proved and the authors intend to employ it in the future for a detailed analysis of ferrofluid magneto-optical properties with regard to other parameters such as particle size, fluid viscosity or surfactant thickness. 


\subsection{Calculation by mixture law}

The above mentioned calculation method is highly time-consuming, so that a detailed calculation of how the attenuation constant evolves during the process of chain formation becomes unapproachable. For this reason, we propose an alternative method based on a mixture law proposed in [22] and successfully applied to ferrofluids [23]. This law provides an expression for the complex effective permittivity of a heterogeneous sample composed of a host material with spheroidal inclusions:

$\varepsilon_{e f f, U}^{*}=\varepsilon_{e}\left(1+x_{U} *\right)$

where

$\boldsymbol{x}_{U}^{*}=\frac{p\left(\varepsilon_{i}^{*}-\varepsilon_{e}\right)}{\varepsilon_{e}+(1-p) N_{U}\left(\varepsilon_{i}^{*}-\varepsilon_{e}\right)}$

In the former expressions, the asterisk denotes a complex magnitude, $\varepsilon_{e f f, U}^{*}$ is the mixture effective permittivity in the $U$ direction $(U=\|$ or $\perp$ , parallel or perpendicular to the polar axis of the spheroids), $\varepsilon_{e}$ and $\varepsilon_{i}^{*}$ are the host and inclusion permittivities, respectively, $\mathrm{p}$ is the volume fraction occupied by the inclusions and $\mathrm{N}_{\mathrm{U}}$ is the spheroid depolarization factor in the $\mathrm{U}$ direction. This factor is related with the polar and equatorial semiaxes $\boldsymbol{a}_{\|}$and $\boldsymbol{a}_{\perp}$ by means of:

$N_{U}=\frac{a_{\|} a_{\perp}^{2}}{2} \int_{0}^{\infty} \frac{d s}{\left(s+a_{\|}^{2}\right)^{1 / 2}\left(s+a_{\perp}^{2}\right)\left(s+a_{U}^{2}\right)}$

As shown in [23], the latter expression is determined by the spheroid aspect ratio $\boldsymbol{A}=\boldsymbol{a}_{\perp} / \boldsymbol{a}_{\|}$.

As it can be seen in Eq. (4), a variation in the shape of the inclusions involves a variation in the mixture equivalent permittivity. In ferrofluids, inclusions are single particles in absence of magnetic field and a certain combination of single particles and chains in presence of a magnetic field. In [23] it is demonstrated that, as chains remain basically oriented parallel to the applied field, the anisotropy in the heterogeneous mixture can be correctly modeled by a medium with equivalent spheroidal inclusions characterized by their aspect ratio.

In order to apply this idea to our simulations, it is necessary to find how to relate the distribution of chains at any given system state with the aspect ratio of its equivalent spheroidal inclusions. Our solution is based on the parameter $\mathrm{K}$, defined in Subsection 2.2. It is easy to find that the length of the average chain is:

$L_{a v}=(K-1) d_{e q}+D$

where $\mathrm{D}$ is the nanoparticle diameter. As a first approximation, the equivalent aspect ratio of a chain should be linked with the quotient between its transversal and longitudinal dimensions, that is to say, D and $\mathrm{L}_{\mathrm{av}}$, respectively. However, a reasonable equivalence must also take into account that chains of similar length but different spacing between particles should have a different equivalent spheroid: the more separation between particles, the more they contribute as individuals to the global behavior of the system or, in other words, the less they contribute to anisotropy. For these reasons, our proposal is to add a correction factor $\beta$ to the average length and taking as equivalent aspect ratio $A_{e q}$ :

$A_{e q}=\frac{D}{L_{a v}^{\prime}}=\frac{D}{\beta(K-1) d_{e q}+D}$

where the $\beta$ factor depends on $d_{e q}$. The term on the right (except the unknown factor $\beta$ ) is obtained from the results in Subsection 2.2 (Fig. 2 ), and the equivalent aspect ratio on the left is obtained by taking the differences in attenuation factor according to the electromagnetic simulation (Fig. 3) into the mixture law [23].

For the particle concentration analysis, the factor $\beta$ is provided from Eq. (8) with every measurement, obtaining a mean value of $\beta=0.117$. We assume that the $\beta$ factor is constant along the whole series, as it expresses the influence of $d_{e q}$ on the mixture anisotropy and $d_{e q}$ remains constant along those calculations $\left(d_{e q}=330 \mathrm{~nm}\right)$. On the other hand, simulations depending on the $d_{e q}$ must consider a different $\beta$ value for each series.

This way, the attenuation constant for light propagation parallel or perpendicular to the applied magnetic field, at any time after its switch on, can be calculated taking into account Fig. 2: the $\mathrm{K}$ values presented lead to $\mathrm{A}_{\mathrm{eq}}$ values in a straightforward way (Eq. (8)). With $\mathrm{A}_{\mathrm{eq}}$, the depolarization factors $\boldsymbol{N}_{\perp}$ and $\boldsymbol{N}_{\| \text {can }}$ be obtained and then the complex effective permittivity in parallel and perpendicular directions to the applied magnetic field. Finally, the attenuation constant changes can be calculated departing from the complex permittivity, as it is well known. By application of this process to every series in Fig. 2, time evolution of the attenuation constant is obtained. Fig. 4 shows the results obtained.

In order to do the latter calculations, it is important to take into account an effective permittivity for internal homogenization of the core-shells nanoparticles to introduce in the mixture law expressions. Since both core and coating sizes are not small enough with respect to the wavelength, sphere effective permittivity approximations in the Literature are not useful. For that reason, we have used an effective index of $2-0.1 \mathrm{i}$ for the coated spheres, a fair value between both particles materials.

The trends obtained in Fig. 4 are in agreement with experimental results [23] and the ones from Subsection 3.1. The variations over the temporal evolutions are due to the low number of particles in the cell (182 particles in the highest concentration), and therefore, the limited statistics when chains are formed during the simulation. These results show that the method to obtain the optical transmission from the chain average length could be useful to reduce the simulation time and to analyze more easily the dependence on several intrinsic parameters involved in the ferrofluid response.

\section{Conclusions}

A numerical method has been developed to predict the ferrofluid optical response to an external magnetic field application. Simulations of ferrofluid particle distribution evolution have been carried out, showing the dependences on intrinsic parameters as particle concentration and surfactant repulsion. The optical transmission behaviors has been analyze as a function of both intrinsic parameters showing up the importance of any minimal change on them. Both simulation methods present positive results comparing to the expected in the laboratory. The complete method will be used to analyze other parameters such as particle size, magnetization, field strength and fluid viscosity. These simulations, compared with the experimental results, will allow us to understand the mechanisms involved in ferrofluids to optimize them for their potential applications.

\section{Acknowledgement}

This work was supported in part by the national program MINECO TEC2014-52642-C2-2-R and in part by the Universidad de Zaragoza UZ2017-CIE-07 and UZ2018-CIE-07.

\section{References}

[1] S. Odenbach, Ferrofluids: Magnetically Controllable Fluids and Their Applications, Berlin: Springer, 2002, doi: 10.1007/3-540-45646-5. 
[2] S. Pu, X. Chen, Y. Chen, Y. Yu, W. Liao, L. Chen, Y. Xia, J. Appl. Phys. 99 (2006), 093516.

[3] Y. Miao, J. Wu, W. Lin, K. Zhang, Y. Yuan, B. Song, Opt. Express 21 (2013) 29914.

[4] Y. Miao, X. Ma, J. Wu, B. Song, H. Zhang, K. Zhang, B. Liu, J. Yao, Opt. Lett. 40 (2015) 3905.

[5] S. Brojabasi, V. Mahendran, B.B. Lahiri, J. Philip, Opt. Commun. 342 (2015) 224.

[6] G.N. Rao, Y.D. Yao, Y.L. Chen, K.T. Wu, J.W. Chen, Phys. Rev. E 72 (2005), 031408.

[7] L. Chen, J. Li, X. Qiu, Y. Lin, X. Liu, H. Miao, J. Fu, Opt. Commun. 316 (2014) 146.

[8] J.M. Laskar, J. Philip, B. Raj, Phys. Rev. E 78 (2008), 031404.

[9] R. Patel, R.V. Upadhyay, R.V. Mehta, J. Magn. Magn. Mater. 300 (2006) E217.

[10] Q. Zhang, Q.J.H. Wang, H.S. Zhu, IEEE Trans. Magn. 32 (1996) 297.

[11] S.I. Shulyma, B.M. Tanygin, V.F. Kovalenko, M.V. Petrychuk, J. Magn. Magn. Mater. 416 (2016) 141.

[12] A. Tasker, R.W. Chantrell, J.J. Miles, M.R. Parker, A. Bradbury, IEEE Trans. Magn. 24 (2) (1988) 1671.

[13] W.C. Elmore, Phys. Rev. 60 (1941) 593

[14] M.T.A. Eloi, J.L. Santos, P.C. Morais, A.F. Bakuzis, Phys. Rev. E 82 (2010), 021407.

[15] J. Gregory, Adv. Colloid Interface Sci. 147-148 (2009) 109.
[16] M. Ivey, J. Liu, L. Zhu, S. Cutillas, Phys. Rev. E 63 (2000), 011403.

[17] S. Brojabasi, T. Muthukumaran, J.M. Laskar, J. Philip, Opt. Commun. 336 (2015) 278.

[18] A.Y. Solovyova, E.A. Elfimova, A.O. Ivanov, P.J. Camp, Phys. Rev. E 96 (2017), 052609.

[19] M. Hassan, A. Zeeshan, A. Majeed, R. Ellahi, J. Magn. Magn. Mater. 443 (2017) 36.

[20] J.G. Yardley, R.C. McPhedran, N.A. Nicorovici, L.C. Botten, Phys. Rev. E 60 (1999) 6068.

[21] S.C. Lee, J. Opt. Soc. Am. A 28 (2011) 1812.

[22] A. Sihvola, J.A. Kong, IEEE Trans. Geosci. Rem. Sens. 26 (1988) 420.

[23] Á. Sanz-Felipe, J.C. Martín, J. Phys. D: Appl. Phys. 51 (2018) https://doi.org/10. 1088/1361-6463/aab05f.

[24] T. Kruse, A. Spanoudaki, R. Pelster, Phys. Rev. B 68 (2003), 054208.

[25] J. Li, X. Liu, Y. Lin, X. Qiu, X. Ma, Y. Huang, J. Phys. D: Appl. Phys. 37 (2004) 3357.

[26] http://www.chembk.com/en/chem/Methyl\%20methacrylate

[27] http://www.chembk.com/en/chem/EGDMA

[28] S. Kedenburg, M. Vieweg, T. Gissibl, H. Giessen, Opt. Mat. Express 2 (2012) 1588.

[29] M.R. Querry, Optical constants Contactor Report CRDC-CR-85034, University of Missouri, 1985 